\title{
A Study on Assessment of Frequency, Intensity, Disability and Severity Associated with Primary Headaches
}

\author{
Tinu Merin Thomas', Bileena K', Preethy Mathew Karanath', Ann Mary Swaroop ${ }^{1 *}$, Srinivas R ${ }^{2}$ \\ ${ }^{1}$ Department of Pharmacy Practice, Faculty of Pharmacy, Ramaiah University of Applied Sciences, M.S.R. Nagar, Bengaluru-54, India \\ ${ }^{2}$ Department of Neurology, M.S. Ramaiah Hospitals, Bengaluru-54, India
}

\begin{tabular}{|c|c|}
\hline ARTICLE INFO & ABSTRACT \\
\hline Article history: & \multirow{5}{*}{$\begin{array}{l}\text { Introduction: Primary headache is one of the major complaints that accounts for more than } 1 \% \text { of emergency visits } \\
\text { worldwide. Currently global prevalence of primary headaches is } 47 \% \text {. However, the disease remains underdiagnosed } \\
\text { and undertreated in a majority of suffering population, inspite of its disabling symptoms. Objective: To assess } \\
\text { the frequency, intensity, disability and severity of primary headaches. Methodology: The study was designed as } \\
\text { a prospective observational study and was carried out for a period of six months. The survey was performed by } \\
\text { means of one to one interview, employing Headache-Attributed Restriction, Disability, Social Handicap and Impaired } \\
\text { Participation (HARDSHIP) questionnaire, Visual Analogue Scale (VAS) and headache diary. After three months, } \\
\text { enrolled volunteers were followed up. Results: Amongst the study population (250), 50.4\% (126) of the individuals } \\
\text { were diagnosed with Tension Type Headache (TTH) and experienced mildly severe, pressing type, bilateral pain. } \\
\text { However, migraine (113) was manifested majorly as throbbing/pulsating pain. Assessment of frequency depicted that } \\
22.4 \%(56) \text { of subjects experienced headache at least for two days in one month. Amongst the migraineurs } 24.7 \%(28) \\
\text { reported an intensity of just bearable pain on the VAS. Conclusion: Quality of life was affected in individuals with } \\
\text { headache due to the negligence of this condition in their lives. There is an immense need to counsel and treat such } \\
\text { individuals as headache significantly affects their lifestyle. }\end{array}$} \\
\hline /09/2017 & \\
\hline & \\
\hline 018 & \\
\hline ICHD Criteria & \\
\hline
\end{tabular}

\section{INTRODUCTION}

Headache disorders are highly under diagnosed or under treated amongst the majority of suffering population, inspite of its disabling symptoms. This may be attributed to the negligence of sufferers in addition to increasing dependency over nonprescription analgesics or consultation limited to outpatient basis. Clinically, physicians face immense difficulties in the assessment of symptoms that limit accurate diagnosis and hinder the facilitation of outpatient care and management in patients suffering from headache (El-Sherbiny et al., 2015). A recent systematic review stated various clinical features to differentiate migraine from nonmigraine headache: pounding headache, unilateral pain associated with nausea, duration of headache lasting for 4 to 72 hours, and headache-related disability (Olesen et al., 2004). Although

\section{${ }^{*}$ Corresponding Author}

Dr. Ann Mary Swaroop, Assistant Professor, Faculty of Pharmacy, MSRUAS, MSRIT Post, Bangalore-560054, India.

E-mail: annmary103@gmail.com extremely frequent, TTH is rarely severe and rarely causes an Emergency Department (ED) visit. It is classically bilateral, pressing or tightening pain with mild to moderate intensity and the pain does not aggravate with physical activity (Lipton et al., 2002). Cluster Headache $(\mathrm{CH})$ is an atypical headache and an infrequent cause of emergency admissions especially in comparison to migraine or TTH (Sjaastad et al., 2003). Hence, there is scarcity in the neuro-epidemiological studies conducted to analyze the impact of $\mathrm{CH}$. $\mathrm{CH}$ presents with excruciating pain generally situated in or around one eye but may radiate to other areas of face, head, neck and shoulder. Similar to migraine, it is unilateral pain with severe to very severe intensity, duration lasting 15 to 180 minutes and is associated with restlessness, excessive tearing and redness in the affected eye. Attacks have a frequency from 1 every other day to 8 per day (Leroux et al., 2008).

More than $1 \%$ of the emergency visits world-wide comprise of primary headache (Dipiro et al., 2012). Migraine, Tension Type Headache (TTH) and Cluster Headache (CH) are 
collectively known as Primary Headache Disorders (Friedman et al., 2009). The current global prevalence of primary headache, migraine and tension type headache (TTH) are 47\%, 10\% and $38 \%$ respectively. The lifetime prevalence rates for headaches are higher in women when compared with men, of which TTH accounts for $88 \%$ (Oshinaike et al., 2014).

The Global Burden of Disease Survey 2013 (GBD2013) established that tension type headache and migraine are among the top 10 causes of disability worldwide and ranking third when measured in years of life lost to disability (YLDs) (Steiner et al., 2015). Although majority of these are established to be benign, they are associated with painful acute exacerbated headache episodes (Friedman et al., 2009).

Numerous studies have assessed the prevalence of headache within the general population, professional groups, and work place settings, however there were fewer studies that focused only on university students. The prevalence of migraine among medical students ranges from 11 to $40 \%$ worldwide (Al-Hashel et al., 2014). The epidemiological studies among medical students are of great interest since previous reports indicate that headaches have peak prevalence during productive ages resulting in more disability and restriction of life that negatively affects the efficacy of these individuals (Al-Hashel et al., 2014, Blaauw et al., 2014). Headache frequency has been noted to be increased during educational years of students as they are subjected to stress with regard to their exams, anticipated higher levels of performance, many years of education and an implicit responsibility to the courses, this causes high risk of suicide and disability (Al-Hashel et al., 2014, Park et al., 2015). Students with headaches tend to miss more school than other students, which inturn may influence the future occupational performance of these students in the society (Al-Hashel et al., 2014) .

A variety of measures are available in the recent years to assess the severity of problems associated with headaches and to weigh their impact on the life of these patients. The frequency and intensity of pain in headache can be measured using patient case reports and from daily headache recordings (Holroyl et al., 1999).

Disability adjusted life years (DALY) was defined as an important indicator of disability due to diseases in patients by the World Health Organization (WHO). Headache disorders are estimated to have a high score of DALY indicating that it is extremely associated with disability. Quantification of the disability in headache patients is of great importance so as to determine the severity of disease and to provide appropriate management (D'Amico et al., 2001).

Lipton et al. 1994 developed the migraine disability assessment (MIDAS) questionnaire to assess various aspects of migraine (Zandifar et al., 2014). Headache Disability Index (HDI) is another measure of great importance. These have been extensively studied and used in clinical practice in many countries. These enable clinicians to identify the patients more distressed by headache and make optimal decision in order to tailor treatment according to their needs. The rationale of this study is to develop comparative data on the frequency, intensity, severity and disability index of different types of Primary headaches using various questionnaires.

\section{METHODOLOGY}

\section{Study site}

The study was carried out at M.S. Ramaiah Dental and Pharmacy College, Bangalore.

\section{Study period}

The study was conducted for a period of 6 months from November 2015 to April 2016.

\section{Study design}

The study conducted was a community based prospective observational study.

\section{Ethical approval}

The study was approved by the Institutional Human Ethical Committee (Reference number: MSRMC/EC/2016).

\section{Study criteria}

\section{Inclusion criteria}

- Patients who had primary headaches for more than 1 year as per ICHD beta version 3 criteria.

- Patients of either gender aged $\geq 18$ years of age.

\section{Exclusion criteria}

- Patients with chronic medical illness such as Stroke, Epilepsy, Brain tumor, Meningitis that might cause secondary headache.

\section{Sources of data}

- Patient interviews

- Questionnaires

Study procedure

The study was carried out in different stages.

Screening

Screening was performed among 440 students and faculties of M.S Ramaiah Dental and Pharmacy colleges. Out of the 440 individuals, 250 fulfilled the inclusion criteria. Participation in the study was completely voluntary.

\section{Pilot survey}

Pilot study was conducted in 30 students randomly selected from the 250 individuals. All the participants were informed about the nature and procedure of the study and their written consent was obtained. The purpose of the pilot survey was to test the questionnaires for their feasibility and to make final adaptations based upon feedback.

\section{Designing of Data collection form \& Data collection}

The survey was performed by means of an interview, using a detailed structured assessment questionnaire. The HARDSHIP questionnaire (Headache-Attributed Restriction, Disability, Social Handicap and Impaired Participation) was adapted from Lifting The Burden (LTB), a UK-registered organization conducting the Global Campaign against Headache. This organization works in official relations with the World Health Organization. The questionnaire is widely accepted and was employed previously in a total of 19 countries in 18 languages including India. 
The questionnaire was composed of personal and demographic enquiry, headache screening questions and diagnostic questions based on the International Classification of Headache Disorders (ICHD-III) beta version. Finally the burden attributed to headache and quality of life among the individuals were also assessed.

Primary headaches were categorized according to the International Classification of Headache Disorders (ICHD) criteria into migraine, TTH and $\mathrm{CH}$. It is accepted as the official classification of headache disorders by World Health Organization (WHO). In our study we used ICHD-III beta version, which is the latest and revised version of ICHD that has improved sensitivity and specificity over the previous ICHD criterias.

Migraine was diagnosed in subjects with recurrent, moderate to severe unilateral throbbing headache associated with nausea or vomiting or visual disturbances.

Tension-type headache was diagnosed when subjects suffered from bilateral or pressure-like feeling in the absence of gastrointestinal or visual discomfort.

Data were collected via face-to-face interview instead of performing a self-administered survey as it is a less sensitive method than a clinical interview.

During the interview, participants' headache frequency (number of days) over the preceding 1 month was recorded. The study participant's headache intensity was recorded using a rating scale, which categorized the intensity as "not bad", "quite bad" and "very bad". The headache intensity ratings were transformed into a numerical scale ranging from 1-3, which was treated as a continuous variable for ease of statistical analysis.

During pilot study, the categorization of headaches was found to be complex, therefore additional questions were asked to gather information about aura symptoms, menstruation and cluster headache symptoms etc., for further categorization of primary headaches. Headache intensity was measured on a four point scale ranging from 0-3 as "no headache", "mild headache", "moderate headache"," severe headache". This scale is recommended for use in headache research by the International Headache Society (IHS).

A list of common triggers was provided in the questionnaire which included the following items: emotional events, stress, anxiety/ depression, noise, exams, reading, eating habits, fasting, menstruation, irregular sleep, physical activity, smoking etc. All the participants were asked to indicate triggers for their headaches.

In addition, all the participants were also provided with headache diary to record their headache episodes for a span of 3 months. During this period, participants were asked to describe the factors that aggravate or diminish the headache episodes from a predetermined list of precipitating factors. Patients had an opportunity to record the additional risk factors during each headache episode.

After 3 months, patients were followed up and the completed headache diaries were obtained from the participants.

\section{Data management}

Completed paper questionnaires and physicians' evaluations were entered into Microsoft Excel and eventually imported into SPSS software for ease of statistical analysis. Paper records are stored securely for further data evaluation.

\section{RESULT}

Among the 250 individuals, $58 \%$ of females (145) and $42 \%$ of males (105) suffered from primary headache. The mean age of the study population was found to be 23 years $(\mathrm{SD} \pm 4.6)$. Out of the total study population, maximum $79.2 \%$ (198) of individuals belonged to the age group of 18-25 years (Table 1). Tension type headache was the most common 55\% (139) primary headache disorder among the population followed by migraine $44 \%$ (113) and cluster headache $1 \%$ (2), among which TTH 55.3\% (77) and migraine 61.9\% (70) were predominantly identified in females. Cluster headache was found only in 2 individuals both of whom were females 1\% (2) (Fig. 1).

Table 1: Age categorization in the study population.

\begin{tabular}{ccc}
\hline Age Group & $\begin{array}{c}\text { Number Of Patients } \\
(\mathbf{N}=\mathbf{2 5 0})\end{array}$ & Percentage (\%) \\
\hline 18-25 years & 198 & 79.2 \\
$26-33$ years & 41 & 16.4 \\
$34-41$ years & 10 & 4 \\
$42-49$ years & 1 & 0.4 \\
\hline
\end{tabular}

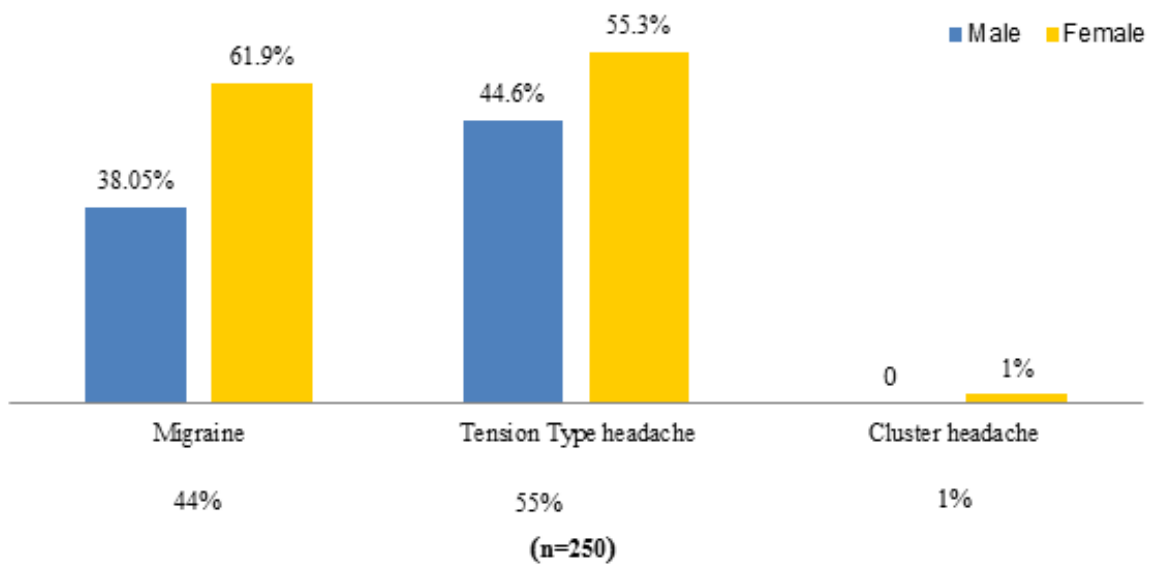

Fig. 1: Type and Gender distribution of Primary Headache in study population. 


\section{Characteristics of primary headache}

Among the 250 individuals who were diagnosed to have primary headache, the quality of pain experienced by $50.4 \%$ (126) was tight/pressing type. Tight/pressing type pain was commonly noted among individuals with tension type headache $(76.2 \%)$ where as throbbing/pulsating pain was commonly seen among individuals with migraine (82.3\%). While categorizing the location of pain it was seen that $78.4 \%$ (109) of the individuals experienced bilateral pain. The most commonly associated symptoms were photophobia and phonophobia which were identified mainly among the individuals diagnosed as migraine. Even though the duration of pain was higher among migraineurs, the interference of headache in their daily activities was found to be low $16.81 \%$ (19) (Table 2).

Table 2: Characteristics of Primary Headache.

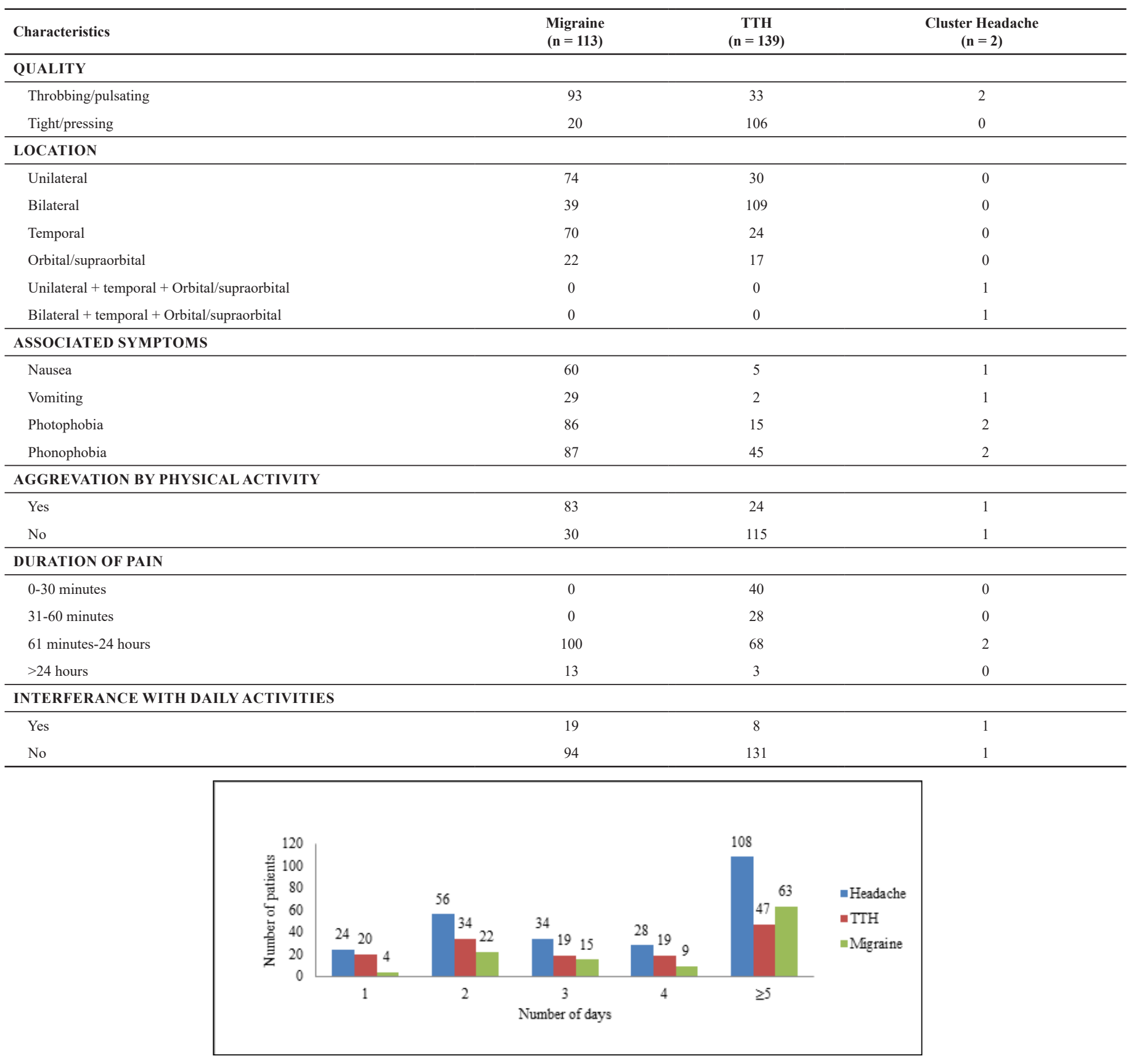

Fig. 2: Frequency of Primary Headache, TTH, Migraine in study population $(n=250)$.

\section{Frequency of headaches in study population}

The frequency of headaches, TTH and migraine among the study population are depicted in Fig. 2. Among the 2 individuals with cluster headache, the frequency of headache was found to be 10 days and 5 days in the past one month. 


\section{Visual analogue scale in study population}

Among the 113 individuals diagnosed with migraine, most of the individuals reported just bearable pain in VAS scale. Among the 139 tension type headache individuals, 21.5\% (30) individuals experienced uncomfortable pain which was followed by moderate pain $15.8 \%$ (22). The severity of pain was found to be less in tension type headache than migraine population (Fig. 3 ). Among the 2 cluster individuals, one had experienced just bearable pain and the other had horrible pain during headache attacks.

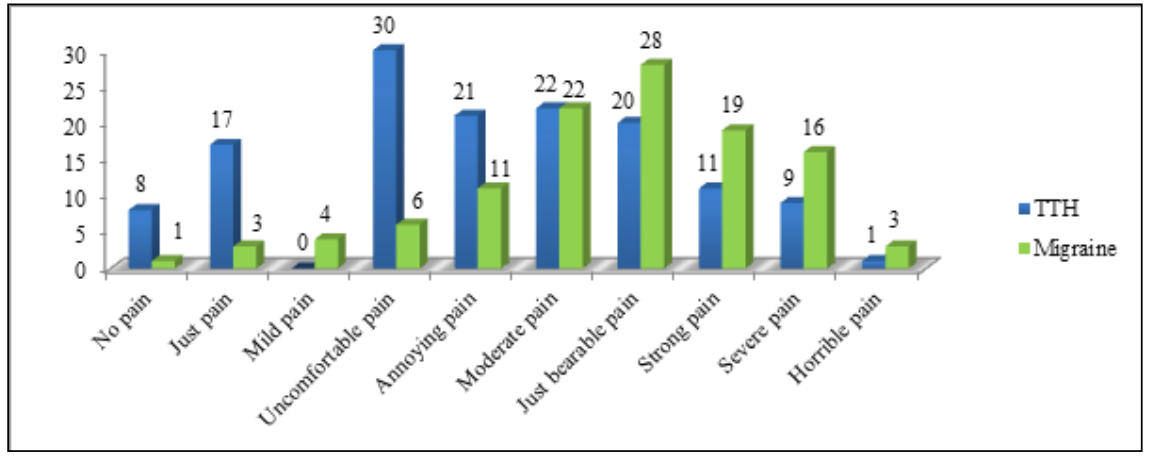

Fig. 3: Visual Analogue Scale in TTH, Migraine population.

\section{Headache disability index in study population}

Among the total population, $41.6 \%$ (104) of the individuals experienced mild disability whereas a few $4.8 \%$ (12) experienced very severe disability due to headache. The study showed that $41.59 \%$ (47) of the migraine individuals experienced mild disability. Of the 139 TTH individuals, $41.72 \%$ (58) experienced mild disability (Fig. 4). Among the 2 individuals with $\mathrm{CH}$, one had experienced mild disability whereas the other experienced very severe disability.

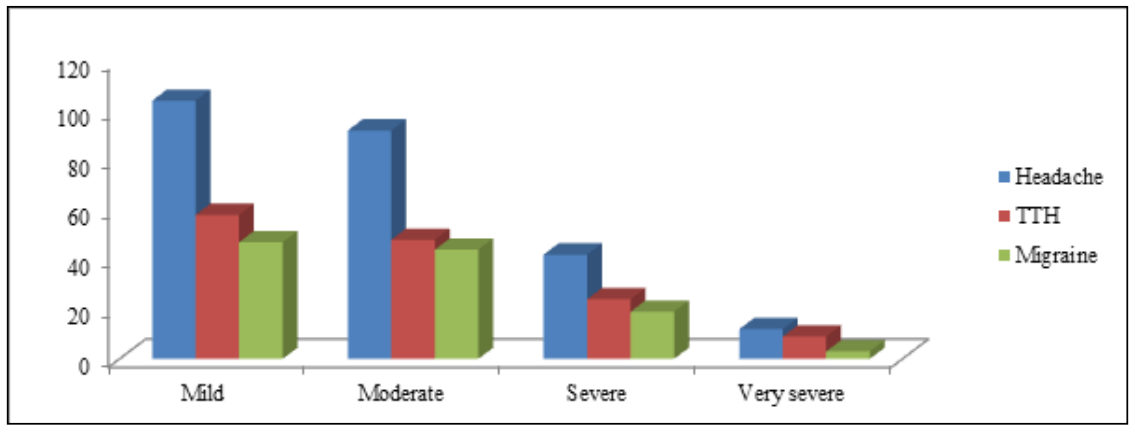

Fig. 4: Headache Disability Index in Primary Headache, Migraine, TTH.

\section{Midas grade in migraine population}

The disability rate among migraine population was high. Individuals who suffered from migraine reported that it significantly interfered with their day to day activities. This was analyzed using MIDAS questionnaire. It was found that $40.7 \%$
(46) of the individuals had severe disability (Grade 4) and 26.54\%

(30) experienced moderate disability (Grade 3 ) due to migraine (Fig. 5). Among the 113 individuals who experienced migraine, higher $54.34 \%$ (25) disability grade was seen among males than females $45.65 \%$ (21). A significant difference was found between men and women with regard to their disability score (Fig. 6).

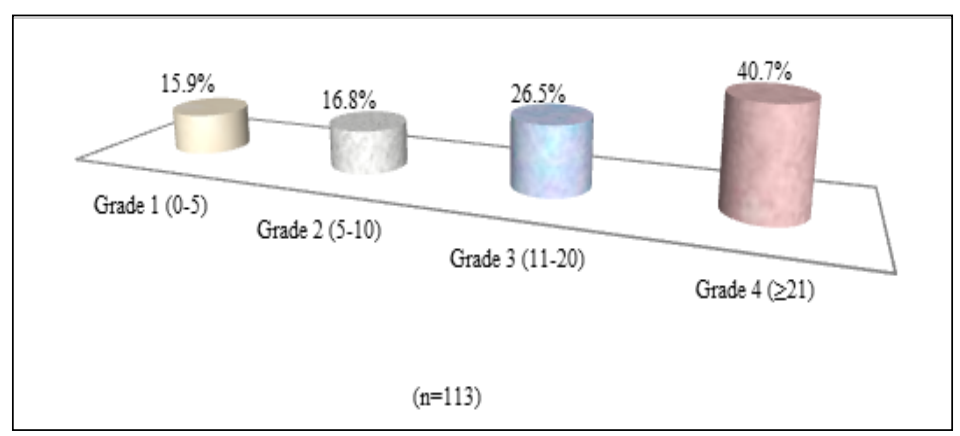

Fig. 5: Midas Grade in Migraine population. 


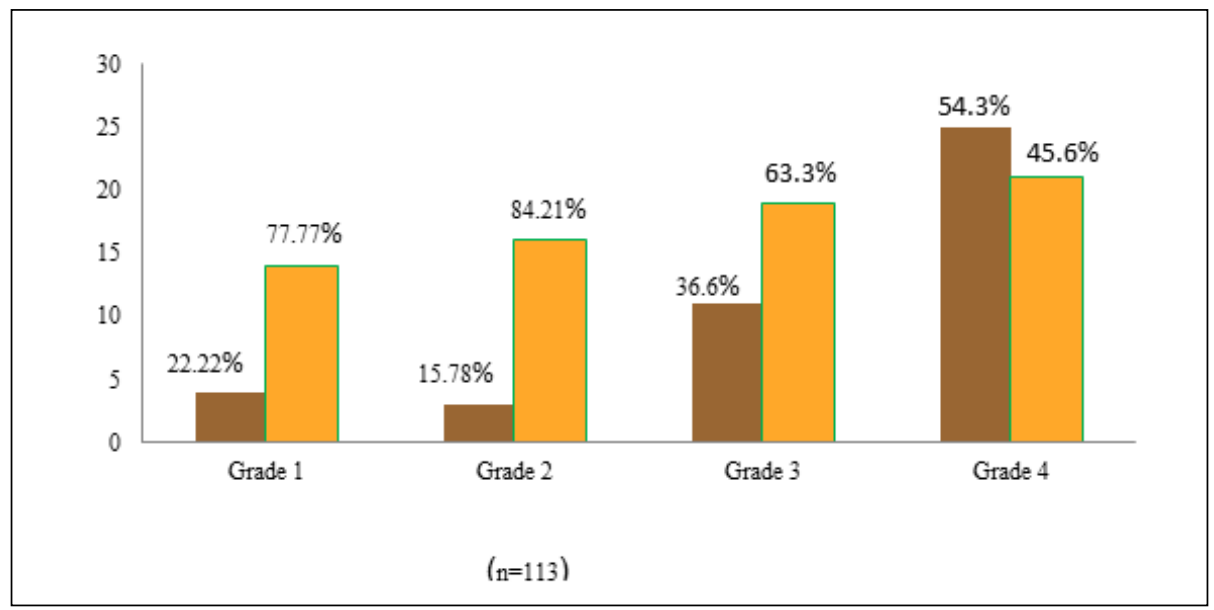

Fig. 6: Gender Distribution in Midas Grade.

\section{DISCUSSION}

The present study was conducted among the students and faculties of M.S Ramaiah Dental and Pharmacy Colleges, Bangalore for a period of 6 months. This study was the first epidemiological survey conducted for the assessment of frequency, intensity, severity and disability of primary headache among this population. Previous studies conducted in India among students have concentrated only to give epidemiological data on headaches.

During the study period a total of 250 cases of primary headache were monitored prospectively. In our study it was identified that the occurrence of primary headache was more common among the age group of $18-25$ years, this might be because most of our study population were students with female predominance (58\%) when compared to males (42\%). Our results were also consistent with a previous study conducted among Indian adolescents where a higher $(60.6 \%)$ headache prevalence was observed among girls (Gupta et al., 2009).

In our study it was identified that the pain characteristics of those individuals with TTH differed from those with other types of primary headache. Among the total population, 50.4\% of the individuals with TTH experienced pressing type pain, which was bilateral in nature and was of mild severity. Throbbing/pulsating pain was commonly seen among individuals with migraine. Almost similar results were observed in study conducted in primary school children in which $61 \%$ of TTH individuals experienced pressing type pain (Galinovic et al., 2009).

In our study it was reported that a large (55.6\%) percentage of individuals suffered from TTH than migraine $(45.2 \%)$. But in controversy migraine was found to be more prevalent than TTH in a study conducted among Indian adolescents (Gupta et al., 2009). This discrepancy may be due to geographical differences, differences in diagnostic criteria as well as the study design. Higher prevalence rates of migraine in females can be associated with hormonal factors (Fukui et al., 2008). In our study cluster headache was identified only in 2 individuals. This might be because in general the occurrences of cluster headaches are rare and infrequent.

Our hypothesis was that owing to high prevalence of tension-type headache, the disability caused by this type of headache would be greater than other types of headache. But it was observed that migraine population experienced a higher (Grade 4) disability when compared with other types of headaches. This higher disability among migraine population might be because patients with TTH are less likely to suffer from disability symptoms such as nausea, vomiting, photophobia and phonophobia. Factors such as exercise and physical activity also do not exacerbate symptoms among the patients with TTH. Similar results were observed in a study conducted in Turkey (Demirkirkan et al., 2006).

In our study it showed that most of the people experienced headache frequency of two days. Migraine also shows similar result with frequency. In controversy, a study conducted in Turkey showed that migraine frequency was five days (Demirkirkan et al., 2006). In our study TTH individuals experienced headache frequency of two days. Similar result were showed in a study conducted in Shiraz (Ayatollahi et al., 2006).

\section{CONCLUSION}

Quality of life is affected in individuals with headache due to negligence of impact of this condition on their life. This study identified that headache led to higher disability and interference in daily activities. There is a great need for addressing this health problem as the frequency, intensity and severity of primary headache was found to be high among the population. Explanation for increased incidence of headache probably lies in lifestyle and/or environmental factors. There is an immense need to counsel and treat such individuals, as headache significantly affects an individual, family and society.

\section{LIMITATION}

- In our study, we included patients with frequent headache episodes (atleast one headache episode in every month). This might have led to the omission of patients with infrequent headache.

- Since majority of the population belonged to the age group of 18-25 years, generalizability of our findings to other populations is limited.

- The study was carried out for a short period of time. 


\section{ABBREVIATIONS}

HARDSHIP, Headache-Attributed Restriction, Disability, Social Handicap and Impaired Participation; VAS, Visual Analogue Scale; ICHD-III, International Classification of Headache Disorders beta version; TTH, Tension Type Headache; ED, Emergency Department; CH, Cluster Headache; GBD2013, The Global Burden of Disease Survey 2013; YLDs, Years of life lost to disability; DALY, Disability adjusted life years; WHO, World Health Organization; MIDAS, Migraine disability assessment; HDI, Headache Disability Index; ICHD, Classification of Headache Disorders.

\section{ACKNOWLEDGEMENT}

We acknowledge Dr. V Madhavan, Dean and Principal, Dr. E. Maheswari, Professor and Head of Department of Pharmacy Practice, Faculty of Pharmacy, M.S. Ramaiah University of Applied Sciences and Dr. Srinivas R, and Mrs. Preethy Mathew Karanath for their continuous support and encouragement.

\section{AUTHORS CONTRIBUTION}

Tinu Merin Thomas and Bileena K collected the data, did the statistical analyses and wrote the first draft of this paper. The study was done under the guidance of Mrs. Preethy Mathew Karanath and Dr. Srinivas R. The paper was edited by Dr. Ann Mary Swaroop for important content. All authors reviewed and provided comments on subsequent iterations.

\section{COMPETING INTEREST}

None of the authors have any competing interest pertaining to the manuscript.

\section{FUNDING SOURCES}

This research did not receive any specific grant from funding agencies in the public, commercial, or not-for-profit sectors.

\section{REFERENCES}

Al-Hashel J, Ahmed S, Alroughani R, Goadsby P. Migraine among medical students in Kuwait University. J Headache Pain, 2014; 15(1):26.

Ayatollahi SMT, Khosravi1A. Prevalence of migraine and tension type headache in primary-school children in Shiraz. East Mediterr Health J, 2006; 12(6): 809-17.

Blaauw B, Dyb G, Hagen K, Holmen T, Linde M, Wentzel-Larsen T, Zwart JA. Anxiety, depression and behavioral problems among adolescents with recurrent headache: the Young-HUNT study. J Headache Pain, 2014; 15(1):38.

D’Amico D, Mosconi P, Genco S, Usai S, Prudenzano AMP, Grazzi L, Leone M, Puca FM, Bussone G. The Migraine Disability Assessment (MIDAS) questionnaire: translation and reliability of the Italian version. Cephalalgia, 2001; 21(10):947-52.

Demirkirkan M, Ellidokuz H, Boluk A. Prevalence and Clinical
Characteristics of Migraine in University Students in Turkey. Tohoku J Exp Med, 2006; 208(1):87-92.

Dipiro JT, Talbert RL, Yee GC, Matzke GR, Wells BG, Posey LM. 2012. Pharmacotherapy: a pathophysiologic approach, 7th. ed. United States: Mc Graw Hill; 1005-19.

El-Sherbiny N, Masoud M, Shalaby N, Shehata H. Prevalence of primary headache disorders in Fayoum Governorate, Egypt. J Headache Pain, 2015; 16(85):1-8.

Friedman B, Grosberg B. Diagnosis and Management of the Primary Headache Disorders in the Emergency Department Setting. Emerg Med Clin North Am. 2009; 27(1):71-87.

Fukui PT, Gonçalves TRT, Strabelli CG et al. Trigger factors in Migraine patients.Arq Neuropsiquiatr, 2008; 66(3a):494-99.

Galinovic I, Vukovic V, Troselj M. Migraine and Tension-Type Headache in Medical Students: A Questionnaire Study. Coll. Antropol, 2009; 33(1): 169-73.

Gupta R, Bhatia MS, Dahiya D, Sharma S, Sapra R, Semalti K, Dua RPS. Recurrent Headache in Indian Adolescents. Indian J Pediatr, 2009; 76 (7):733-37.

Holroyl KA, Malinoski P, Davis MK, Lipchik GL. The Three Dimensions of Headache Impact:Pain,Disability and Affective Distress;Pain, 1999; 83(3);571-78.

Leroux E, Ducros A. Cluster headache. Orphanet J Rare Dis. $2008 ; 3(20)$.

Lipton RB, Scher AI, Kolodner K, Liberman J, Steiner TJ, Stewart WF. Migraine in the United States: epidemiology and patterns of health care use. Neurology, 2002; 58(6):885-94.

Olesen, Jes, Steiner TJ. The International classification of headache disorders, 2nd edn (ICDH-II), 2004; 808-11.

Oshinaike O, Ojo O, Okubadejo N, Ojelabi O, Dada A. Primary headache disorders at a tertiary health facility in lagos, nigeria: prevalence and consultation patterns. BioMed Research International, 2014; 1-5.

Park S-P, Seo J-G, Lee W-K. Osmophobia and allodynia are critical factors for suicidality in patients with migraine. J Headache Pain, 2015; 16(1).

Sjaastad O, Bakketeig LS. Cluster headache prevalence.Vaga study of headache epidemiology. Cephalalgia, 2003; 23(7):528-33.

Steiner TJ, Birbeck GL, Jensen RH, Katsarava Z, Stovner LJ, Martelletti P. Headache disorders are third cause of disability worldwide. J Headache Pain, 2015; 58(16);1-3.

Zandifar A, Asgari F, Haghdoost F, Masjedi SS, Manouchehri N Banihashemi M, Ghorbani A, Najafi MR, Saadatnia M, Lipton RB. Reliability and Validity of the Migraine Disability Assessment Scale among Migraine and Tension Type Headache in Iranian Patients. BioMed Research International, 2014; 1-7.

How to cite this article:

Thomas TM, Bileena K, Karanath PM, Swaroop AM, Srinivas R, A Study on Assessment of Frequency, Intensity, Disability And Severity Associated with Primary Headaches. J App Pharm Sci, 2018; 8(02): 112-118. 